\title{
USING DESIS AND EO-1 HYPERION REFLECTANCE TIME SERIES FOR THE ASSESSMENT OF VEGETATION TRAITS AND GROSS PRIMARY PRODUCTION (GPP)
}

\author{
P. E. K. Campbell ${ }^{1,2}$, K. F. Huemmrich ${ }^{1,2}$, E. M. Middleton ${ }^{2}$, J. Alfieri ${ }^{3}$, C. van $\operatorname{der}^{\text {Tol }}{ }^{4}$, C. S. R. Neigh ${ }^{2}$ \\ ${ }^{1}$ Joint Center for Earth Systems Technology, University of Maryland Baltimore County, Baltimore, MD 21228, USA - (petya, \\ huemmric)@umbc.edu; ${ }^{2}$ NASA Goddard Space Flight Center, Greenbelt, MD 20771, USA - (elizabeth.m.middleton, \\ christopher.s.neigh)@ nasa.gov; ${ }^{3}$ USDA-ARS Hydrology and Remote Sensing Laboratory, Beltsville, MD 20705, USA - \\ (Joe.Alfieri)@ars.usda.gov; ${ }^{4}$ ITC, University of Twente, Enschede, NL - (c.vandertol)@ utwente.nl
}

\section{Commission I, WG I/1}

\begin{abstract}
KEY WORDS: DESIS, EO-1 Hyperion, surface reflectance, leaf total chlorophyll content (Cab), eddy covariance observations, gross primary production (GPP), partial least squares regression (PLSR), Soil Canopy Observation Photosynthesis Energy model (SCOPE), canopy leaf area index (LAI), canopy chlorophyll (Cab*LAI), vegetation function.
\end{abstract}

\begin{abstract}
:
This study evaluates the potential of the DLR Earth Sensing Imaging Spectrometer (DESIS) visible through near-infrared (VNIR) surface reflectance to augment the EO-1 Hyperion full spectrum (400-2400 nm) reflectance collection over vegetated flux sites, to extend the reflectance time series up to the present. We compared DESIS and Hyperion surface reflectance magnitude and variability at a pseudo-invariant site (PICS) and a vegetated flux site (VFS). VNIR reflectance magnitudes between the two sensors did not significantly differ at the PICS. However, DESIS variability was higher, likely due to differences in the data acquisition time and observation geometry. Using empirical and biophysical models, both DESIS and Hyperion datasets captured the seasonal variations in gross primary production (GPP) and canopy bio-physical parameters such as chlorophyll content, leaf area index (LAI), and senescent material at the VFS. Differences in the magnitudes of the bio-physical parameters were observed, likely due to the differences in the sensors spectral range and resolution. Using together VNIR reflectance from EO-1 Hyperion and DESIS convolved to Hyperion spectral resolution to estimate canopy chlorophyll and GPP, we demonstrate that combining historic and current spacebased reflectance data in a common multi-sensor approach is feasible. This is of importance for extending the reflectance record established with EO-1 Hyperion to provide continuity with the current orbital instruments (e.g., DESIS/ISS, PRISMA/ASI) and the forthcoming NASA Surface Biology and Geology (SBG), ESA CHIME and DLR EnMAP satellite missions, which is of key importance for comparisons of current and past trends in the seasonal dynamics of vegetation traits and photosynthetic function.
\end{abstract}

\section{INTRODUCTION AND BACKGROUND}

With the changing climate, it has become critical to understand the dynamics in vegetation function, as the ecosystems respond to variable environmental conditions and cycle through the seasonal changes (Jones and Vaughan, 2010). Plant spectral traits are increasingly recognized as key to understanding ecosystems adaptation to environmental changes and recently the studies of plant function and vegetation traits using hyperspectral remote sensing have significantly increased (Zhang et al., 2020). The ability to monitor vegetation traits and carbon accumulation is of great interest when comparing productivity across seasons and among vegetation types. Longterm data records of corresponding orbital, and vegetation field and eddy covariance observations are required for such environmental monitoring and change detection.

Multi-date and multi-sensor satellite environmental monitoring requires consistency and radiometric stability of the data, to carry out biophysical parameter surveys that are of sufficient sensitivity and accuracy and are reproducible over time. The incoming surface radiance is subject to several factors which influence the stability and sensitivity of the reflected vegetation signal captured from space, including suboptimal sensor signal to noise ratio, deficiencies in the information and algorithms utilized in data calibration and processing, as well as variation in the accuracy of removal of atmospheric absorption and scattering effects to derive surface reflectance from top of atmosphere (TOA) radiances (Gao et al., 2009). While atmospheric correction accounts for the differences in solar illumination during the year at various locations and daily acquisitions time, it also introduces variations in the reflectance data, due to differences between the modelled and real atmospheres and the implemented approaches (Gao et al., 2009). As a result, the cumulative error in the reflectance signal may lead to a variation of several percent in each spectral band, which could limit the sensitivity of the data, and/or introduce significant differences and apparent spectral trends.

Reflectance, the spectral fraction of light reflected by a surface, is an essential Earth observation variable, provides the basis for developing products such as vegetation cover, chlorophyll content and productivity (Badawi et al., 2019; Burggraaf, 2020). High spectral resolution reflectance $(\leq 10 \mathrm{~nm})$ provides an efficient tool for synoptic evaluation of the ability of vegetation to sequester carbon and reflect radiation, due to changes in vegetation chemical and structural composition. Remote sensing is rapidly advancing beyond the classical multi-spectral approaches (Schimel et al., 2019) and new instruments are now providing higher spectral, spatial, and temporal resolution reflectance data, offering the potential for improved ability to describe vegetation function and canopy bio-physical traits.

The German Aerospace Center (DLR) Earth Sensing Imaging Spectrometer (DESIS) on the International Space Station (ISS) currently provides hyperspectral images, offering potential for evaluation of vegetation pigment content, photosynthetic capacity, and detection of vegetation stress. The collection of 
DESIS images over flux sites started in 2019, and currently at some of the sites there are image collections capturing vegetation reflectance and canopy traits at multiple growth stages across the seasons. Recently, the DESIS surface reflectance product became available, covering the visible and near-infrared region (VNIR, 400-1000 $\mathrm{nm}$ ) at $2.5 \mathrm{~nm}$ spectral and $30 \mathrm{~m}$ spatial resolutions.

The Earth Observing-1 (EO-1) Hyperion spectrometer collected more than 10,000 images over flux sites during its operation (i.e., 2001-2017), which provides a consistent spectral record capturing vegetation traits across multiple seasons and years. Hyperion spectra cover the visible-shortwave infrared (VSWIR) $(400-2500 \mathrm{~nm})$ range at $\sim 10 \mathrm{~nm}$ spectral and $30 \mathrm{~m}$ spatial resolution (Middleton et al., 2013). The use of Hyperion surface reflectance data to estimate canopy constituents including nitrogen and chlorophyll content, biophysical properties such as leaf area index (LAI) and canopy closure, and to characterize vegetation function and gross primary production (GPP) is well documented (Martin et al., 2008; Olinger et al., 2008; Zheng et al., 2008; Gershman et al., 2009; Asner et al., 2008). The new DESIS reflectance collection offers the potential to augment the Hyperion collection for flux sites, if at semi-invariant targets the data compare without significant differences in spectral magnitude and variability.

The aim of this study was to evaluate the potential of using DESIS to augment the existing EO-1 Hyperion reflectance collection for flux sites, to thus extend the time series for assessment of vegetation photosynthetic potential and function. Our objectives were to: 1 ) evaluate the magnitude and stability of the DESIS surface reflectance data, as compared to Hyperion reflectance at a semi-invariant test site, the Railroad Valley Playa (RRVP); and 2) compare the use of DESIS and Hyperion surface reflectance data with empirical and integrated biophysical and radiative transfer models (RTM) to characterize the seasonal changes in vegetation traits and GPP at a vegetated flux site, the United States Department of Agriculture (USDA) Optimizing Production Inputs for Economic and Environmental Enhancement (OPE3).

The use of empirical and integrated biophysical and RTM models with hyperspectral imagery is an accepted, physically sound approach to retrieve the bio-physical variables associated with spectral differences because the approaches are generic and therefore generally applicable (Ustin \& Gamon, 2010).

\subsection{Study Sites}

The RRVP Pseudo-Invariant Calibration Site (PICS) in Nevada, approximately 300 miles north of Las Vegas and 100 miles east of Tonopah, has a dry climate typical for the high deserts of western USA. Because of its large size, RRVP is used for evaluation of sensors with large footprints (Thome et al., 2003). The surface layer of the site is relatively smooth and spatially homogeneous, consisting of compacted clay-rich lacustrine deposits, however the site suffers from the presence of iron absorption features, which is typical for the playas in this region (Telliet et al., 2007).

The vegetated OPE3 flux site at the USDA Agricultural Research Service in Beltsville, Maryland, is a 22 ha production field, which is heavily instrumented and belongs to the Long Term Agro-ecosystem Research (LTAR) network of sites. At OPE3 a $10 \mathrm{~m}$ tall flux tower is set up in a cornfield which is planted annually. The local climate is warm and temperate, with hot, humid summers, and typically mild winters with occasional freezes, which provide a strong variation in seasonal leaf area index and canopy chlorophyll patterns.

Detailed descriptions of both study sites and a list of relevant citations are available in Campbell et al. (2013).

\subsection{Data Collections}

The spectral and spatial characteristics of the orbital data and products are described in detail in the DESIS Algorithms Theoretical Basis Document (DESIS ATBD, 2015) and the EO-1 User's Manual (2003). EO-1 flew in a polar orbit at $\sim 700 \mathrm{~km}$ and at flux sites collected images between 9:30-10:30 am, at $\leq$ 20 degrees off nadir. The DESIS nominal altitude is $400 \mathrm{~km}$, however the observation times and viewing geometry widely vary, due to the oblique orbit of the ISS. The images used for this study were free of clouds and shadows, collected $\leq 15$ degrees of nadir and within \pm 2 hours of noon local (EST).

The DESIS images (11 images for RRVP and 10 for OPE3) were downloaded, using the Teledyne Brown Engineering Geospatial Solutions data distribution system (TCloud, https://teledyne.tcloudhost.com/), as surface reflectance covering the VNIR spectral range with 187 bands at $2.5 \mathrm{~nm}$ spectral and $30 \mathrm{~m}$ spatial resolution (L2A product). The L2A VNIR product was especially suitable for convolution of the 2.5 $\mathrm{nm}$ DESIS bands to the $10 \mathrm{~nm}$ Hyperion VNIR bands.

The Hyperion VNIR-SWIR (VSWIR) images were downloaded as calibrated at-sensor radiances (L1R, 242 bands, 196 of which are well calibrated) from the USGS EarthExplorer (http://earthexplorer.usgs.gov/) and processed using the Atmosphere CORrection Now (ACORN, 2008) software to derive VSWIR surface reflectance, as described in Campbell et al. (2013). Band subsetting was used to remove uncalibrated and overlapping bands, and bands with low signal to noise ratio (SNR) and high variability adjacent to water absorption features, resulting in subsets of 170 bands, covering portions of the VSWIR region.

Eddy covariance data collected in 2000-2020 at the OPE3 vegetation site, were available for use in the study. Net Ecosystem Production (NEP), a measure of the net carbon accumulation in an ecosystem, is determined as the difference between GPP and ecosystem respiration $(\mathrm{Re})$. The flux tower at OPE3 is equipped with sonic anemometers mounted above the vegetation canopy, which measure NEP using eddy covariance methods (Baldocchi, 2008). Daytime Re was calculated from relationships developed between nighttime NEP and air temperature, and GPP was computed as the sum of NEP and Re (Reichstein et al., 2005). Midday flux averages were calculated over a four-hour period, centering on noon (EST), and analyzed in combination with averaged spectra from the hyperspectral imagery on the same day.

Field data available for different growth conditions in the 20082017 growing seasons, include both leaf and canopy measurements. Leaf measurements include total chlorophyl (Cab), photosynthetic light curves, fresh and dry weight, reflectance and transmittance. Canopy measurements include leaf area index (LAI), canopy Cab (Cab*LAI), height, canescent fraction, etc. They were paired with the Hyperion collection from the corresponding date for further analysis. Flux data for 2000-2020 were paired by date of acquisition with the spaceborne images. 


\subsection{Data processing and analysis}

We identified the location of the RRVP calibration site and the OPE3 flux tower footprint on the images. Local spatial statistics (i.e., Getis Ord statistics and Moran's I index, ENVI 2020) were used to discern spatially homogeneous areas, and regions of interest (ROIs) were defined for spectral extraction. We collected spectra from 10 to 20 Hyperion and DESIS pixels within the ROIs, which were averaged to produce reflectance means per date, and to calculate the associated standard deviations and coefficients of variation (Tab. 1, Fig. 1, Fig. 2). Thus, we obtained two reflectance datasets per site: DESIS VNIR at 2.5 $\mathrm{nm}$, and Hyperion VSWIR at $10 \mathrm{~nm}$. To produce a combined extended reflectance dataset, we merged the VNIR DESIS and Hyperion reflectance data. We subsetted the Hyperion data to the DESIS region. Further, the DESIS and Hyperion sensor response functions were used to convolve the DESIS bands to the Hyperion bands as a weighted average of the signal at each band, to thus produce extended time series of spectrally comparable datasets.

Field GPP, Cab, LAI and other field measurements were paired by date with the Hyperion and DESIS reflectance datasets and used to: 1) produce statistical Partial Last Squares regression (PLSR, Singh et al., 2015) models; and 2) parameterize and implement the biophysical model Soil Canopy Observation Photosynthesis Energy (SCOPE, van der Tol et al., 2009; 2014).

PLSR models are routinely used with hyperspectral reflectance to estimate canopy traits including GPP (Martin et al., 2008; Singh et al., 2015). We used the approach and routines for deriving PLSRs described in Singh et al. (2015). However, the development of robust PLSR requires the use of a significant number of paired field traits (e.g., GPP, Cab*LAI) and reflectance $(25+$, Singh et al., 2015). However, we had 26 Hyperion and 10 DESIS images. Therefore, we derived PLSR models using: 1) Hyperion VSWIR data alone; and 2) the combined Hyperion+DESIS VNIR dataset. We were not able to produce PLSRs using only the original $2.5 \mathrm{~nm}$ DESIS data.

The SCOPE model is an integrated 1-D model, which combines plant physiology, radiative transfer, and micrometeorology models, to link top of canopy observations of radiance and reflectance with land surface processes such as photosynthesis, net radiation, sensible and latent heat flux, fluorescence (not used here) and soil heat flux. We implemented SCOPE v. 1.73, which consists of leaf and canopy biophysical and radiative transfer modules, which can be used together and/or inverted independently. In a prior study we parameterized the biophysical leaf model using representative OPE3 field data and estimated the maximum carboxylation capacities (Vcmo, umol m-2 s-1) and photosynthetic efficiencies for C3 and C4 crops (van der Tol et al., 2014). Here, we implemented the canopy RTMo in an inversion, using mean reflectance per date from Hyperion, DESIS (Fig. 3) and the convolved Hyperion+DESIS datasets, to estimate the canopy traits for the dates of image collection. The RTMo iteratively optimizes the trait estimates, maximizing the correlation $\left(\mathrm{R}^{2}\right)$ and minimizing RMSE between measured and simulated canopy reflectance. The RMSE and $\mathrm{R}^{2}$ are reported to compare the performance of the inversions and the derived traits. To initialize the RTMo and for evaluation of the derived traits, we used field measurements of total chlorophyll (Cab), leaf area index (LAI) and GPP. The traits derived with the RTMo inversion include: chlorophyll content (Cab ug.cm-2), dry matter content (Cdm g.cm-2), leaf water thickness equivalent $(\mathrm{Cw} \mathrm{cm})$, senescent material $(\mathrm{Cs}, 0$ 1), carotenoids (Ccs ug.cm-2), leaf structure parameter (N), leaf area index [LAI m2.m-2], leaf inclination (LIDF -1/1). To

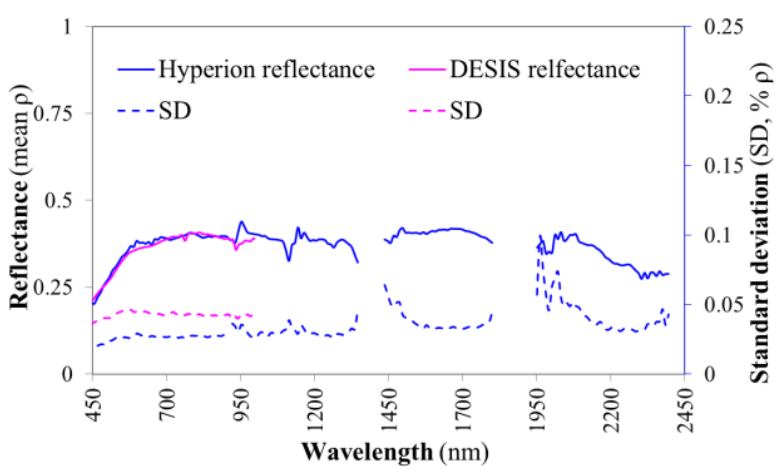

Figure 1. Mean reflectance magnitude (solid lines) and variation (SD, dashed lines) at Railroad Valley Playa (RRVP) as measured by Hyperion $(\mathrm{n}=15,10 \mathrm{~nm}$, blue) and DESIS $(\mathrm{n}=11,2.5 \mathrm{~nm}$, pink).

compare the use of the RTMo in the inversions with the different reflectance datasets, we calculated the means, standard deviations, ranges, and coefficients of variation for key vegetation traits (Tab. 2). To implement SCOPE 1.73 in a forward simulation of GPP, we used the derived traits and specified the parameters below, based on field measurements at OPE3. In the implementation we specified Vcmo (range 25-40 umol m-2 s-1; van der Tol et al., 2014), air temperature (range 5-32 $\mathrm{C}^{\circ}$ ), average annual temperature $\left(26 \mathrm{C}^{\circ}\right)$, broadband incoming shortwave radiation (range 550-650 W m-2), and measurement height (constant $10 \mathrm{~m}$ ). For all other inputs we maintained the default values provided with the model. The estimated GPP was compared to flux measurements, calculating RMSE and $\mathrm{R}^{2}$ for the prediction.

Analysis of variances and descriptive statistics were used to evaluate the temporal and spectral variability of the data. The resulting means, standard deviations (SD) and coefficients of variation $(\mathrm{CV}=$ mean $/ \mathrm{SD}, \%)$ are reported and compared among data types to characterize the temporal variation at each band (Tab. 1 and 2).

\section{RESULTS}

\subsection{Surface reflectance comparison and cross- calibration at the RRVP calibration site}

The RRVP site is characterized by high reflectance values across a wide VSWIR spectral range (Fig.1).

Table 1. Comparing for Railroad Valley Playa (RRVP) Hyperion and DESIS reflectance means, standard deviations and coefficients of variation for select wavelengths.

\begin{tabular}{ccccccc}
\hline \multirow{2}{*}{ Wavelength } & \multicolumn{3}{c}{ Railroad Valley Playa $($ RRVP), NV, USA } \\
\cline { 2 - 7 } (nm) & \multicolumn{3}{c}{ Hyperion Reflectance } & \multicolumn{4}{c}{ DESIS Reflectance } \\
\cline { 2 - 7 } & Mean & SD & CV & Mean & SD & CV \\
\hline 447 & 0.20 & 0.019 & 0.093 & 0.21 & 0.036 & 0.089 \\
549 & 0.34 & 0.025 & 0.074 & 0.32 & 0.045 & 0.078 \\
651 & 0.37 & 0.027 & 0.074 & 0.37 & 0.043 & 0.074 \\
854 & 0.39 & 0.028 & 0.071 & 0.40 & 0.042 & 0.069 \\
1003 & 0.40 & 0.026 & 0.065 & & & \\
1679 & 0.42 & 0.033 & 0.079 & & & \\
2204 & 0.32 & 0.034 & 0.105 & & & \\
\hline
\end{tabular}



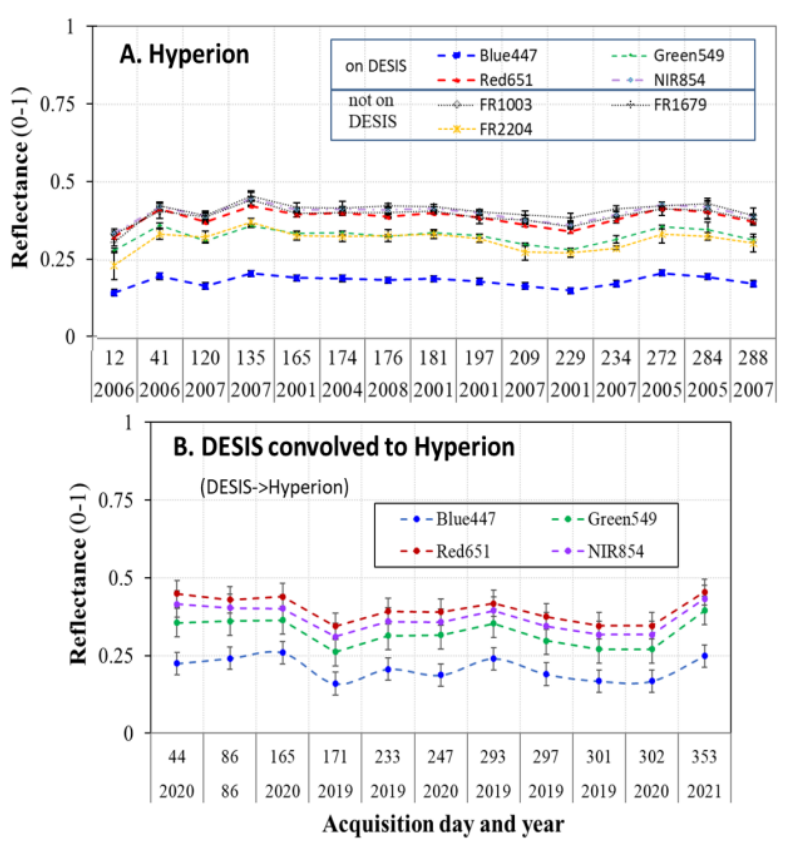

Figure 2. Temporal variation in the reflectance at the RRVP, for select bands: A. Hyperion VSWIR $10 \mathrm{~nm}$ reflectance, and B. DESIS VNIR $2.5 \mathrm{~nm}$ reflectance convolved to Hyperion 10 $\mathrm{nm}$ resolution (DESIS $\rightarrow$ Hyperion) (means, $\pm \mathrm{SD}, \mathrm{n}=15$ ).

The mean reflectance signals of Hyperion and DESIS compared well in the VNIR region (Fig. 1). However, the DESIS signal exhibited higher SD and CVs (Fig. 1, Tab. 1). The temporal variation in the combined VNIR Hyperion with the convolved DESIS data are represented using key spectral bands (Figs. 2, A \& B). During the winter months both datasets had higher SDs, which were possibly due to imperfections in the atmospheric corrections. The magnitude of the mean Hyperion and DESIS reflectance per spectral band did not statistically differ. However, the Hyperion VNIR data exhibited a more uniform and flat temporal profile with lower SDs (Fig. 2).

Both Hyperion and DESIS data were devoid of radiometric temporal and spectral trends, which provides sufficient confidence in the ability of the reflectance data to describe the temporal dynamics at the OPE3 site. The Hyperion and the convolved DESIS datasets were strongly corelated $\left(\mathrm{R}^{2}=0.98\right)$ in the 447-905 $\mathrm{nm}$ region (Fig. 3). However, in wavelengths below $447 \mathrm{~nm}$ and above $905 \mathrm{~nm}$ the convolved DESIS reflectance underestimated the Hyperion values, which is possibly due to

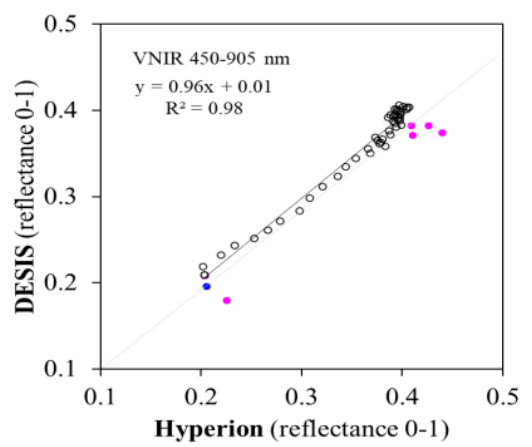

Figure 3. Comparison of the VNIR mean reflectance signals of Hyperion and DESIS convolved to Hyperion bands. The VNIR bands compared well in the 447-905 nm region (in black). The DESIS bands below $447 \mathrm{~nm}$ and above $905 \mathrm{~nm}$ (in pink) were excluded from further analysis. the diferent atmospheric correction approaches. These bands were excluded from the analysis.

\subsection{Seasonal variation in the Hyperion and DESIS canopy reflectance at the $\mathrm{OPE} 3$ vegetated flux site}

At OPE3, both the Hyperion and DESIS reflectance displayed typical vegetation seasonal trends (Fig. $4 \mathrm{~A}$ and $\mathrm{B}$ ). We observed relatively low VIS values due to high absorption by leaf photosynthetic pigment content, but relatively hight NIR values due to weak absorption and strong scattering by canopy structural components. However, the magnitude of the DESIS reflectance data was significantly higher in the NIR as compared to Hyperion (Fig. 4, A vs. B). Since we established at RRVP that the magnitude of DESIS and Hyperion reflectance compares well, this was not attributed to radiometric differences in the data but was likely due to a significant change in the vegetation and growing conditions at OPE3, where in 2018 a crop rotation to soybean was implemented to improve corn productivity. Hyperion's reflectance in the SWIR (Fig. 4 A, 1450-2450 nm) was relatively low during the growing season, due to absorption by leaf water, cellulose, lignin, and other canopy constituents, causing vegetation to appear relatively dark. Subsequently, the crop appeared brighter during senescence and the harvesting period, due to contributions from the dry vegetation residue and soils.

\subsection{Using Hyperion and DESIS data to implement multi-sensor models for estimating vegetation traits and GPP}

We implemented the Partial Least Square Regression (PLSR) statistical approach and the integrated biophysical model SCOPE (SCOPE v 1.73), two common approaches using high spectral resolution data, to estimate the seasonal variation in key traits and canopy GPP. We compared the PLSR results obtained using the VSWIR Hyperion time series alone to the results from combined VNIR DESIS+Hyperion dataset (Figs. 5 and 6). The PLSR approach requires the use of a minimum of 25

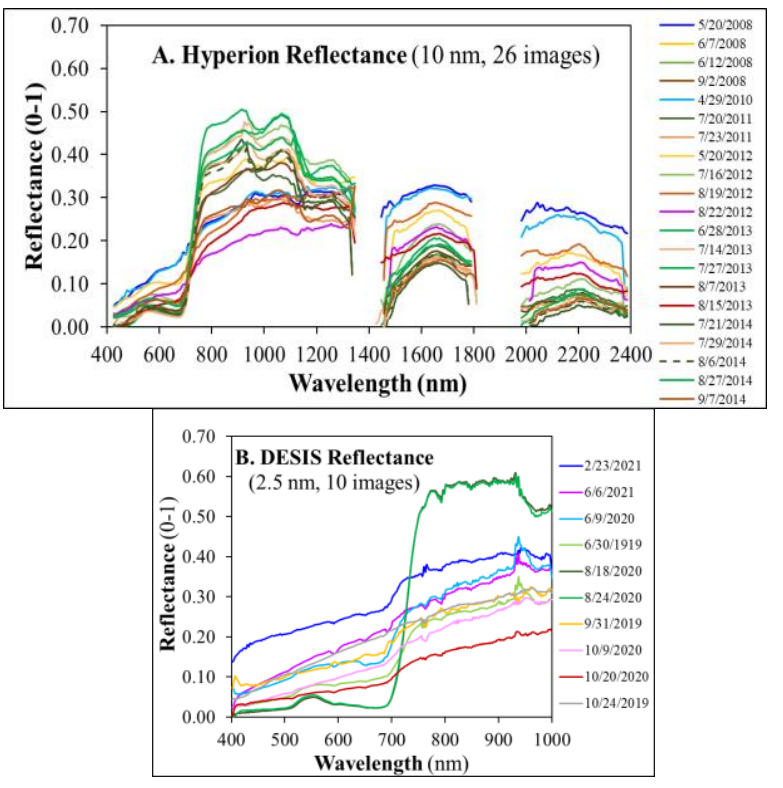

Figure 4. Seasonal variation in reflectance spectra at OPE3 as captured by Hyperion (A. VSWIR $n=26$ ) and DESIS (B. VNIR $\mathrm{n}=10$ ) data. 
observations for developing reliable models, which were not available from DESIS for OPE3. PLSR model performance was evaluated averaging 500 random models with a 50/50 calibration/validation split and reported based on the PLSR regression coefficient. The model's standardized PLSR coefficients (Fig. 6) were used to report the magnitude and direction of influence of each spectral region in the model predictions. Comparing the PLSR predicted vs. observed GPP using Hyperion (Fig. 5 A), the model estimates had a correlation coefficient of $\mathrm{R}^{2}=0.96$ and $\mathrm{RMSE}=2.60$. The predicted residual sum of squares statistic (PRESS, $\pm 1 \mathrm{SD}$ ) was used to determine the number of PLS components, minimizing both PRESS means and SD (after Sing et al., 2015). The final Hyperion GPP PLSR model used seven components. The regions with highest influence on the predictions (Fig. $6 \mathrm{~A}$ ) were the red-edge (1), the NIR around $1200 \mathrm{~nm} \mathrm{(2)} \mathrm{and} \mathrm{the} \mathrm{SWIR} \mathrm{near} 2200 \mathrm{~nm}$ (3). Similar success was achieved for the VNIR Hyperion + DESIS GPP PLSR model (Fig. 5 B) performed with $\mathrm{R}^{2}=0.97$ for predicted vs. observed GPP with RMSE $=2.17$. Two PLS components were determined as optimal, and the regions with highest influence for the predictions (Fig. 6 B) were the shoulders of the green peak ( 1 and 2 ) and the red edge region (3). However, the predicted GPP overestimated the observed values, at the higher range (Fig. 6 B).

The SCOPE model was implemented in two steps: 1) using the original Hyperion and DESIS datasets, and the combined VNIR data (e.g., Hyperion+DESIS) with the RTMo in an inversion to derive biophysical traits; and 2) using the derived traits augmented with additional field data to estimate GPP. A comparison of a subset of the derived traits important for photosynthetic function is provided in Tab. 2. The combined dataset produced the widest range in trait variation and had the

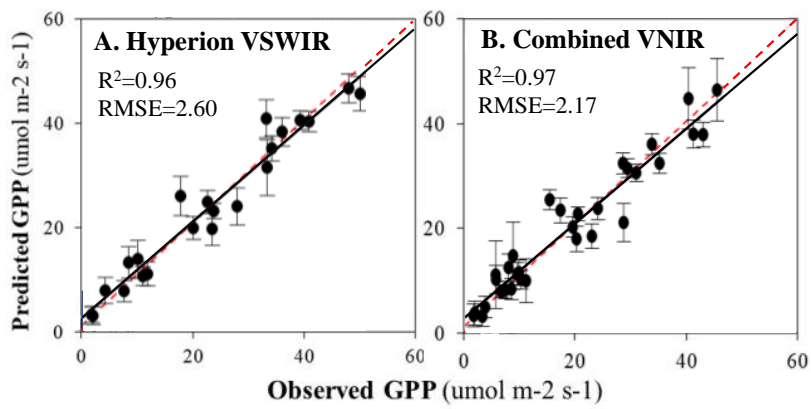

Figure 5. Predicted versus observed gross primary production (GPP) derived using partial least square regression (PLSR) with Hyperion (A. VSWIR, $n=26$ ) and the combined VNIR (B. Hyperion+DESIS, $n=10$ ) reflectance datasets.

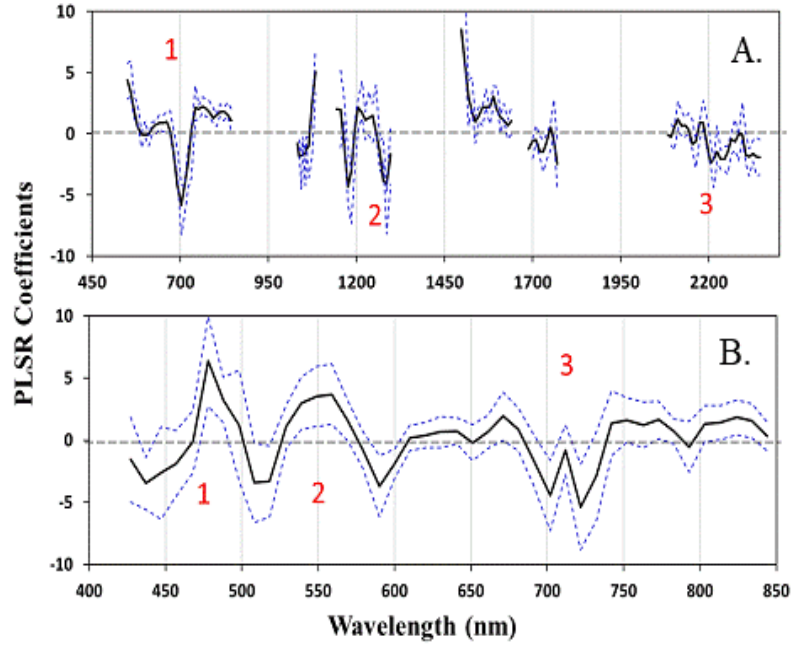

Figure 6. Standardized PLSR coefficients (means solid line, SD dashed lines) for GPP derived using Hyperion (A.) and the combined Hyperion VNIR and DESIS convolved to Hyperion (B.) reflectance datasets. The spectral regions of importance for the predictions are numbered.

lowest average RMSE $=0.011$. The estimates of total chlorophyll $(\mathrm{Cab})$, senescent material (Cs), leaf area index (LAI), and carotenoids (Cca) derived with DESIS $2.5 \mathrm{~nm}$ and DESIS convolved $(10 \mathrm{~nm})$ data were strongly related $\left(\mathrm{Cab} \mathrm{R}^{2}=0.99\right.$, Cs $\mathrm{R}^{2}=0.94$, LAI $\mathrm{R}^{2}=0.77$, Cca $\mathrm{R}^{2}=0.73$ ). However, in the lower range of the traits, the estimates derived with the coarse $10 \mathrm{~nm}$ resolution data were significantly higher than the trait values obtained with the original high spectral resolution DESIS data (Fig. 7). We have observed a similar trend, when upscaling of Cab estimates from higher $(\leq 5 \mathrm{~m})$ to medium $(\leq 30 \mathrm{~m})$ spatial resolution. The order of parameter importance for the predictions was different for the VSWIR and VNIR datasets (Tab. 2). Using the VSWIR Hyperion data, the Cs parameter was first in order of importance for the predictions, followed by dry matter $(\mathrm{Cdm})$, water content $(\mathrm{Cw}), \mathrm{Cab}$ and $\mathrm{Cca}$. Using the VNIR dataset, the most important parameters for the predictions were $\mathrm{Cab}$, Cca and Cs (Tab. 2); while the model provided estimates of $\mathrm{Cdm}$ and $\mathrm{Cw}$, their accuracy was significantly lower using the VNIR spectra.

Using the existing field data for OPE3, we validated the RTMo Hyperion estimates (Tab. 2). Only leaf chlorophyll content $(\mathrm{Cab})$ and leaf area index (LAI) were retrieved with acceptable accuracy and were strongly correlated to the field data (i.e., Cab $\mathrm{R}^{2}=0.82$, and Cab*LAI $\mathrm{R}^{2}=0.77$ ). However, leaf and canopy

Table 2. Subset of key canopy traits derived using the canopy radiative transfer module (RTMo) of SCOPE with Hyperion, DESIS and the combined reflectance datasets (mean, range, coefficient of variation (CV) and order (\#) of importance for the prediction). The accuracy of the prediction is evaluated in terms of root mean square error (RMSE) statistics.

\begin{tabular}{|c|c|c|c|c|c|c|c|c|c|c|c|c|c|c|c|c|}
\hline \multirow{2}{*}{\multicolumn{2}{|c|}{ Canopy Bio-physical Parameters }} & \multicolumn{5}{|c|}{ Hyperion VSWIR } & \multicolumn{5}{|c|}{ DESIS original resolution } & \multicolumn{5}{|c|}{ VNIR Hyperion+DESIS convolved } \\
\hline & & \multicolumn{2}{|c|}{ Range } & \multirow{2}{*}{ Mean } & \multirow{2}{*}{ SD } & \multirow{2}{*}{ CV \# } & \multicolumn{2}{|c|}{ Range } & \multirow{2}{*}{ Mean } & \multirow{2}{*}{ SD } & \multirow{2}{*}{ CV \# } & \multicolumn{2}{|c|}{ Range } & \multirow{2}{*}{ Mean } & \multirow{2}{*}{ SD } & \multirow{2}{*}{ CV \# } \\
\hline traits & units & $\min$ & $\max$ & & & & $\min$ & $\max$ & & & & $\min$ & $\max$ & & & \\
\hline Total chlorophyll & $\mathrm{Cab}\left(\mu \mathrm{g} / \mathrm{cm}^{2}\right.$ & 0.79 & 53.31 & 25.0 & 19.45 & 0.784 & 0.14 & 50.80 & 11.7 & 20.25 & 1.731 & 0.26 & 61.20 & 23.7 & 22.27 & 0.941 \\
\hline Water content & $\mathrm{Cw}\left(\mathrm{g} / \mathrm{cm}^{2}\right)$ & 0.0001 & 0.0291 & 0.014 & 0.01 & 0.643 & 0.0001 & 0.0073 & 0.004 & 0.002 & 0.59 & 0.0002 & 0.0095 & 0.006 & 0.054 & 0.31 \\
\hline Dry matter & $\mathrm{Cdm}\left(\mathrm{g} / \mathrm{cm}^{2}\right)$ & 0.002 & 0.013 & 0.0 & 0.00 & 0.632 & 0.012 & 0.019 & 0.016 & 0.002 & 0.16 & 0.017 & 0.023 & 0.019 & 0.008 & 0.06 \\
\hline Senescent material & Cs (a.u) & 0.16 & 0.40 & 0.3 & 0.08 & 0.251 & 0.042 & 0.40 & 0.3 & 0.15 & 0.473 & 0.046 & 0.40 & 0.3 & 0.11 & 0.353 \\
\hline Carotenoids & $\mathrm{Cca}\left(\mu \mathrm{g} / \mathrm{cm}^{2}\right.$ & 0.78 & 10.52 & 7.3 & 3.24 & 0.445 & 0.025 & 11.00 & 3.6 & 4.42 & 1.242 & 0.001 & 10.19 & 6.5 & 4.21 & 0.652 \\
\hline Leaf Area Index & LAI & 1.05 & 4.8 & 3.0 & 1.3 & 0.44 & 1.94 & 4.71 & 3.3 & 0.9 & 0.26 & 1.52 & 5.11 & 2.2 & 2.8 & 1.26 \\
\hline \multicolumn{2}{|c|}{ Root mean square erro $R M S E$} & & & 0.015 & & & & & 0.012 & & & & & 0.011 & & \\
\hline
\end{tabular}



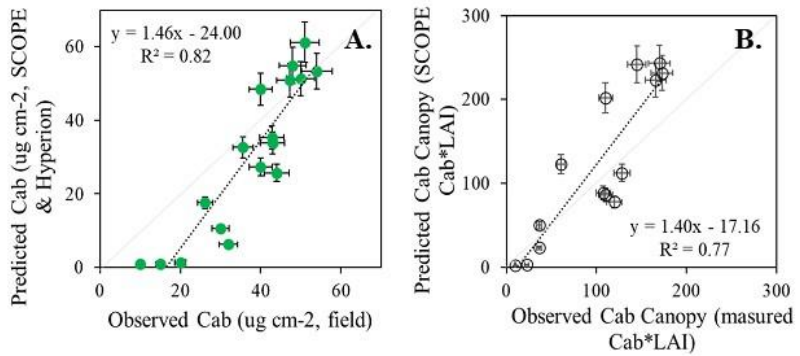

Figure 7. Predicted versus observed leaf total chlorophyll (Cab, A.) and canopy Cab (Cab*LAI, B.), obtained using the SCOPE canopy RTMo in an inversion with Hyperion VSWIR reflectance. Table 2 lists the range and variation of derived traits.

Cab are key drivers of photosynthesis, which allowed us to proceed further using SCOPE 1.73 to estimate mean daily GPP fluxes (Fig. 8). There was a strong correlation between observed

and estimated GPP when using the traits obtained from Hyperion (Fig. 8 A) with $\mathrm{R}^{2}=0.84$ and mean RMSE $=0.015$ when inverting the RTMo to estimate canopy traits. However, the Hyperion estimates slightly overestimated the higher values observed (Fig' 8 A). The GPP estimates derived with traits estimated using DESIS strongly underestimated maximum GPP as compared to the observed values (Fig. 8 B), 55 vs. 28 umol. $\mathrm{m}-2 \mathrm{~s}-1$.

Comparing canopy traits derived using DESIS $2.5 \mathrm{~nm}$ versus 10 $\mathrm{nm}$ reflectance, we established strong relationships for canopy chlorophyll (Cab), senescent material (Cs), LAI and carotenoids (Cca) (Tab. 3). Comparing canopy traits derived using Hyperion's VSWIR versus VNIR reflectance we obtained similarly strong correlations between estimates of $\mathrm{Cab}, \mathrm{Cca}$, LAI and Cs (Tab. 3). Using only VNIR reflectance did not permit reliable estimation of canopy dry matter and/or water content, since the relationships between predicted and observed values were not statistically significant (Tab. 3, marked as ns).

We estimated GPP using the VSWIR Hyperion dataset with both PLSR and SCOPE models and obtained estimates which compared well to the observed values (Fig. 9). However, the Hyperion GPP estimates from SCOPE had lower $\mathrm{R}^{2}=0.84$ and as compared to these obtained using the PLSR model $\mathrm{R}^{2}=0.96$ (Fig. 9)

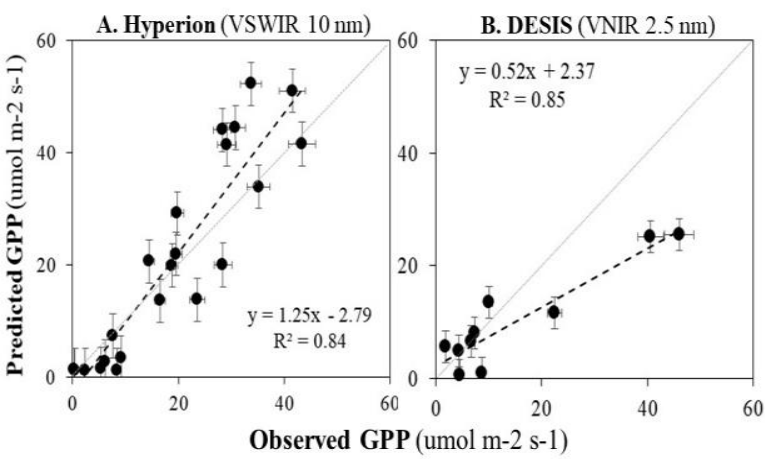

Figure 8. Gross primary production (GPP) estimates derived by SCOPE using A. Hyperion VSWIR data, and B. DESIS data at original resolution (VNIR $2.5 \mathrm{~nm}$ ).
Table 3. Correlation $\left(\mathrm{R}^{2}\right)$ between canopy traits derived using the original DESIS and Hyperion reflectance, versus DESIS $10 \mathrm{~nm}$ resolution and Hyperion VNIR range.

\begin{tabular}{|c|c|c|c|c|}
\hline \multicolumn{2}{|c|}{ Canopy Bio-physical Traits } & $\begin{array}{c}\text { DESIS }^{1} 2.5 \mathrm{~nm} \\
\text { vs. } 10 \mathrm{~nm} \\
\mathrm{R}^{2} \\
\end{array}$ & $\begin{array}{c}\text { Hyperion }^{2} \\
\text { VSWIR vs. VNIR } \\
\mathrm{R}^{2}\end{array}$ & \multirow{2}{*}{$\begin{array}{c}\text { PRISMA }^{2} \\
\text { VSWIR vs.VNI } \\
\mathrm{R}^{2} \\
0.97\end{array}$} \\
\hline Total chlorophyll & $\mathrm{Cab}\left(\mu \mathrm{g} / \mathrm{cm}^{2}\right)$ & 0.99 & 0.96 & \\
\hline Water content & $\mathrm{Cw}\left(\mu \mathrm{g} / \mathrm{cm}^{2}\right)$ & 0.55 & ns & 0.57 \\
\hline Dry matter & $\mathrm{Cdm}\left(\mathrm{g} / \mathrm{cm}^{2}\right)$ & ns & ns & ns \\
\hline Senescent material & Cs (a.u) & 0.94 & 0.59 & 0.94 \\
\hline Carotenoids & $\mathrm{Cca}\left(\mu \mathrm{g} / \mathrm{cm}^{2}\right)$ & 0.73 & 0.92 & 0.96 \\
\hline Leaf Area Index & LAI & 0.77 & 0.82 & 0.97 \\
\hline
\end{tabular}

Currently, work is in progress to process the field Cab and LAI measurements collected in 2019-2021, which will enable us to validate to field data the biophysical parameters, derived through inverting the RTMo with DESIS high resolution and DESIS convolved to Hyperion's $10 \mathrm{~nm}$ data.

\section{DISCUSSION AND CONCLUSIONS}

At the RRVP site, DESIS and Hyperion VNIR reflectance in the 450-905 nm region compared well, providing confidence that these data can be used together in multi-sensor time series. DESIS reflectance had larger variation $(\mathrm{CV})$, which can be improved if the data are normalized for differences in acquisition geometry and time of day for individual collection.

Using the combined VNIR multi-sensor (DESIS+Hyperion) reflectance dataset, we derived PLSR GPP models with higher $\mathrm{R}^{2}$ and lower RMSE as compared to the use of Hyperion VSWIR data alone. By implementing SCOPE with the multisensor dataset, we derived canopy traits for the 2008-2020 timeperiod, which covered a wider range with lower RMSEs for the inversions. Comparing trait estimates using the original DESIS and the convolved DESIS data, while the traits were strongly related (Tab. 3), the lower resolution $10 \mathrm{~nm}$ data over-estimated traits such as LAI and senescent material (Cs).

We estimated GPP using the VSWIR Hyperion dataset with both PLSR and SCOPE (Fig. 9) and obtained estimates which compared well to the observed values, however the PLSR GPP model produced more accurate predictions with lower RMSE and higher $\mathrm{R}^{2}$. The spectral regions sensitive to the changes in photosynthetic pigments were found to have high importance for the PLSR predictions.

Our results demonstrate that the combined use of surface reflectance data from historic and new orbital spectrometers in multi-sensor time series is feasible, providing potential for consistent long-term monitoring of both pseudo-invariant and vegetated sites. Using both empirical and biophysical models, such multi-sensor time series enable the intercomparison of historic and current canopy traits and function.

As additional DESIS, flux and field data become available, we will: 1) extend this analysis to additional vegetation flux sites; 2) augment the multi-sensor time-series for OPE3; 3) confirm the PLSR GPP and SCOPE trait estimates; and 4) validate the estimates against field data. 


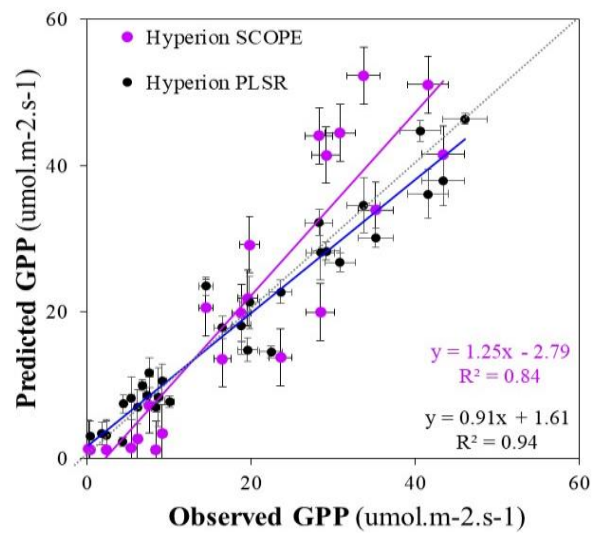

Figure 9. Comparing GPP estimates derived using Hyperion surface reflectance with empirical (i.e., PLSR, black) and radiative transfer and biophysical models (i.e., SCOPE, violet).

In the further effort, we will compare the use of SCOPE and PLSR to estimate vegetation traits versus the use of vegetation features and traditional VIs, to provide a link to the long term Harmonized Landsat-8 Sentinel-2 (HLS) dataset, which will support new product development.

The biophysical properties obtained with imaging spectroscopy could be directly integrated to reduce the uncertainty in ecological and process models (Rogers, Medlyn et al., 2017) to inform model predictions (Fer et al., 2021), thereby reducing current predictive uncertainties, since trait models built at a certain season or year may only be applicable to a limited timeframe and site conditions. Long term consistent reflectance time series are required to monitor the seasonal dynamics in vegetation traits and photosynthetic function to intercompare current and past trends and patterns. Our approach described herein advances the methods for multi-sensor vegetation monitoring and supports the development of a virtual constellation. The constellation of forthcoming spectroscopy missions, such as SBG and others (e.g., PACE, CHIME, DESIS, EnMAP), hold a great potential to address these goals, by facilitating the development of multi-sensor time-series that capture trait dynamics at multiple times per season (CawseNicholson et al., 2021), and bridge the gap to historic datasets (e.g., EO-1 Hyperion), for trait comparison across multiple seasons and years.

\section{ACKNOWLEDGEMENTS}

We gratefully acknowledge the support of the SBG GSFC team, NASA ROSES 17-LCLUC17-0013 and NNH20ZDA001NCSDA grants, which funded this study. This research was a contribution from the Long-Term Agroecosystem Research (LTAR) network. LTAR is supported by the United States Department of Agriculture.

\section{REFERENCES}

Asner, G. P. 2008. Hyperspectral remote sensing of canopy chemistry, physiology and diversity in tropical rainforests," in Hyperspectral Remote Sensing of Tropical and Subtropical Forests, M. Kalacska and G. A. Sanchez-Azofeifa, Eds. New York: Taylor and Francis, 2008, ch. 12.
Atmosphere CORrection Now (ACORN v. 6.1b), (c) 2004-08 ImSpec LLC. Analytical Imaging and Geophysics LLC (AIG), (2001), ACORN User's Guide, Stand Alone Version, 64 p.

Badawi, M., Helder, D., Leigh, L. and Jing, X. 2019. Methods for earth-observing satellite surface reflectance validation. Remote Sens. 11(13), 1543 (2019).

Baldocchi, D., 2008.'Breathing' of the terrestrial biosphere: lessons learned from a global network of carbon dioxide flux measurement systems. TURNER REVIEW No. 15, Australian Journal of Botany, 56 (1), 1-26, 2008.

Burggraaf, O. 2020. Biases from incorrect reflectance convolution. Optics express. Vol. 28, No. 9 / 27 April 2020 / Optics Express 13801. https://doi.org/10.1364/OE.391470

Campbell, P.K.E., E. Middleton, F. Huemmrich, R. F. Kokaly, A. Singh, N. Brunsell, D. Landis \& S. Voorhis (2022). EO-1 Hyperion reflectance time series for assessment of the dynamics in vegetation function, spectral properties and carbon flux assimilation. In preparation

Cawse-Nicholson, K., P. A. Townsend, D. Schimel, A. M. Assiri, P. L. Blake, M. F. Buongiorno, P. Campbell, et al. (50+ co-authors) 2021. NASA's surface biology and geology designated observable: A perspective on surface imaging algorithms. Remote Sensing of Environment, 257: 112349 [10.1016/j.rse.2021.112349]

DESIS ATBD, 2015: DESIS ATBD L1A, L1B, L1C, L2A Processors. Doc. ID PAV-DLR-TN-004, Issue 1.3, 1-85, Date 2015-07-204.

ENVI, Environment for Visualizing Images, Image Processing Software, ver. 5, 2020. L3HARRIS GEOSPATIAL, www.l3harrisgeospatial.com/Software-Technology/ENVI.

Fer, I. et al., 2021. Beyond ecosystem modelling: A roadmap to community cyberinfrastructure for ecological data-model integration. Glob. Change Biol. 2021; 27:13-26. Doi: $10.111 / \mathrm{gcb} .15409$.

Gao, B.-C., Montes, M. J., Davis, C. O. and Goetz, A. F. H. 2009. Atmospheric correction algorithms for hyperspectral remote sensing data of land and ocean. Remote Sens. Environ., vol. 113, pp. S17-S24, 2009, (Supplement 1).

H. G. Jones and R. A. Vaughan, Remote Sensing of Vegetation: Principles, Techniques and Applications. New York: Oxford University Press, 2010, ISBN 978-0-19-920779-4.

Martin, M. E., Plourde, L. C., Ollinger, S. V., Smith, M.-L. and McNeil, B. E. 2008. A generalizable method for remote sensing of canopy nitrogen across a wide range of forest ecosystems. Remote Sens. Environ., vol. 112, no. 9, pp. 3511-3519, 2008.

Middleton, E. M., Ungar, S. G., Mandl, D. J., Ong, L., Campbell, P. K. E., Landis, D. R. , Young, J. P., Pollack, N. H. 2013. The Earth Observing One (EO-1) Satellite Mission: Over a Decade in Space. IEEE Journal of Selected Topics in Applied Earth Observations and Remote Sensing 04/2013; 6(2):243.

Ollinger, S. V. 2011. Sources of variability in canopy reflectance and the convergent properties of plants. New Phytologist, vol. 189, pp. 375-394, 2011.

Reichstein, M. et al., 2005. On the separation of net ecosystem exchange into assimilation and ecosystem respiration: Review and improved algorithm. Global Change Biol., vol. 11, no. 9, pp. 1424-1439, 2005. 
Rodgers, A., Medlyn, B.E., Dukes, J.S., Bonan, G., Caemmerer, S., Dietze, M., Kattage, J., Leakey, A., Mercado, L. M., Niinemets, U. Prentice, C. I., Serbin, S. P., Stich, S., Way, D., and Zaehle, S. 2017. A roadmap for improving therepresentation of photosynthesisin Earth system models. New Phytologist, 213: 22-42.

Singh, A., Serbin, S.P., McNeil, B. E., Kingdon, C. C. and Townsend, P. A. 2015. Imaging spectroscopy algorithms for mapping canopy foliar chemical and morphological traits and their uncertainties. Ecological Applications, 25(8), 2015, pp. 2180-2197.

SYSTAT 12," SYSTAT Software, Inc., San Jose, CA, USA, 20012.

Teillet, P. M., Barsi, J. A. , Chander, G. and Thome, K. J. 2007. Prime candidate earth targets for the post-launch radiometric calibration of space- based optical imaging instruments. Proc. SPIE Conf. 6677 on Earth Observing Systems, vol. XII (2007) Society of Photo-optical Instrumentation Engineers, San Diego, CA.

Thome, K. J., Biggar, S. F. and Wisniewski, W. T. 2003. Crosscomparison of EO-1 sensors and other earth resources sensors to Landsat-7 ETM+ using Railroad Valley Playa. IEEE Trans. Geosci. Remote Sens., vol. 41, pp. 1180-1188, 2003.

van der Tol, C., Verhoef, W. \& Rosema, A., 2009. A model for chlorophyll fluorescence and photosynthesis at leaf scale. Agricultural and forest meteorology. 149, 1, p. 96-105

van der Tol, C., Berry, J. A., Campbell, P. K. E. \& Rascher, U., 2014. Models of fluorescence and photosynthesis for interpreting measurements of solar-induced chlorophyll fluorescence. Journal of geophysical research: Biogeosciences. 119,12 , p. 2312-2327. 